\title{
Tradespace Exploration of Distributed Propulsors for Advanced On-Demand Mobility Concepts
}

\author{
Nicholas K. Borer ${ }^{1}$ and Mark D. Moore ${ }^{2}$ \\ NASA Langley Research Center, Hampton, Virginia 23681 \\ and \\ Andrew R. Turnbull ${ }^{3}$ \\ Analytical Mechanics Associates, Inc. Hampton, VA 23681
}

\begin{abstract}
Combustion-based sources of shaft power tend to significantly penalize distributed propulsion concepts, but electric motors represent an opportunity to advance the use of integrated distributed propulsion on an aircraft. This enables use of propellers in nontraditional, non-thrust-centric applications, including wing lift augmentation, through propeller slipstream acceleration from distributed leading edge propellers, as well as wingtip cruise propulsors. Developing propellers for these applications challenges long-held constraints within propeller design, such as the notion of optimizing for maximum propulsive efficiency, or the use of constant-speed propellers for high-performance aircraft. This paper explores the design space of fixed-pitch propellers for use as (1) lift augmentation when distributed about a wing's leading edge, and (2) as fixed-pitch cruise propellers with significant thrust at reduced tip speeds for takeoff. A methodology is developed for evaluating the high-level trades for these types of propellers and is applied to the exploration of a NASA Distributed Electric Propulsion concept. The results show that the leading edge propellers have very high solidity and pitch well outside of the empirical database, and that the cruise propellers can be operated over a wide RPM range to ensure that thrust can still be produced at takeoff without the need for a pitch change mechanism. To minimize noise exposure to observers on the ground, both the leading edge and cruise propellers are designed for low tip-speed operation during takeoff, climb, and approach.
\end{abstract}

\section{Nomenclature}

$\begin{array}{llll}a_{H} & \text { Coefficient for } 2^{\text {nd }} \text { order term, inner chord } & r & \text { Radial blade location } \\ a_{T} & \text { Coefficient for } 2^{\text {nd }} \text { order term, outer chord } & r_{h u b} & \text { Radial location of blade hub } \\ a_{\beta} & \text { Coefficient for } 2^{\text {nd }} \text { order term, twist } & R & \text { Blade tip radius } \\ A F & \text { Activity factor } & T & \text { Thrust } \\ b_{H} & \text { Coefficient for linear term, inner chord } & \vec{v} & \text { Vector of design parameters } \\ b_{T} & \text { Coefficient for linear term, outer chord } & V & \text { Velocity } \\ b_{\beta} & \text { Coefficient for linear order term, twist } & \bar{V}_{i} & \text { Average total induced velocity from propeller } \\ c & \text { Propeller chord } & V_{t i p} & \text { Propeller tip speed } \\ c_{H} & \text { Constant term, inner chord } & w_{i} & \text { Relative importance of } i^{\text {th }} \text { objective } \\ c_{T} & \text { Constant term, outer chord } & x & \text { Normalized radial blade location, } r / R \\ c_{\beta} & \text { Constant term, twist } & x_{h u b} & \text { Normalized propeller hub location } \\ C_{L_{d e s}} & \text { Design lift coefficient } & x_{H} & \text { Normalized location of maximum chord } \\ C_{L_{i}} & \text { Integrated lift coefficient } & y & \text { Normalized chord value, } c / R \\ C_{P} & \text { Power coefficient } & y_{H} & \text { Ratio of normalized chord at } x=0 \text { to } y_{k} \\ C_{T} & \text { Thrust coefficient } & y_{K} & \text { Normalized maximum chord }\end{array}$

${ }^{1}$ Aerospace Engineer, Aeronautics Systems Analysis Branch, 1 N. Dryden St. MS 442, AIAA Senior Member.

${ }^{2}$ Aerospace Engineer, Aeronautics Systems Analysis Branch, 1 N. Dryden St. MS 442, AIAA Member.

${ }^{3}$ Aerospace Engineer, Aeronautics Systems Analysis Branch, 1 N. Dryden St. MS 442. 


$\begin{array}{llll}D & \text { Propeller diameter } & y_{T} & \text { Ratio of normalized chord at } x=1 \text { to } y_{k} \\ \text { DEP } & \text { Distributed electric propulsion } & \beta & \text { Propeller twist relative to plane of rotation } \\ F & \text { Composite objective function } & \beta_{H} & \text { Propeller twist at } x=0 \\ F_{i} & \text { Value of } i^{\text {th }} \text { objective } & \beta_{J} & \text { Propeller twist at } x=0.5 \\ F_{i}^{*} & \text { Best value of } i^{\text {th }} \text { objective } & \beta_{T} & \text { Propeller twist at } x=1 \\ F_{i}^{-} & \text {Worst value of } i^{\text {th }} \text { objective } & \beta_{3 / 4} & \text { Propeller twist at } 75 \% \text { radius } \\ J & \text { Advance ratio } & \Delta V_{A} & \text { Change in axial velocity behind propeller } \\ m & \text { Number of objectives } & \Delta \bar{V}_{A} & \text { Average change in axial velocity } \\ n & \text { Propeller rotational rate } & \Delta V_{T} & \text { Change in tangential velocity behind propeller } \\ \text { ODM } & \text { On-demand mobility } & \Delta \bar{V}_{T} & \text { Average change in tangential velocity } \\ p & \text { Compromise programming exponent } & \eta & \text { Propulsive efficiency } \\ P & \text { Power } & \bar{\theta} & \text { Average slipstream swirl angle } \\ \bar{q}_{s} / q & \text { Average dynamic pressure ratio } & \rho & \text { Atmospheric density }\end{array}$

\section{Introduction}

Electric propulsion has the potential to offer new degrees of freedom in aircraft performance characteristics and design options. The power and efficiency characteristics of electric motors challenge some of the long-standing constraints that have existed for propellers powered by combustion-based systems, and this necessitates a fresh look at the design and integration of electrically-driven propellers for future aircraft. Future propellers may not just act as efficient thrust-producing devices, but rather as devices to accelerate the flow over the wing to allow the use of smaller, more efficient wings in cruise. This paper seeks to establish a method for evaluating and designing electrically-driven propellers, identify candidate metrics, and highlight some of the salient trades and design choices for concepts enabled by the new degrees of freedom available to electrically-driven propellers.

\section{A. Heuristics for Propeller Design}

Propellers are older than aviation itself, and the means to design and analyze them have evolved through centuries of experimentation and research. The first powered aircraft used propellers to generate thrust, and propeller-driven aircraft are produced to this day in great numbers. Propellers persist because they are a highly efficient means to convert mechanical shaft work into thrust when immersed in a working fluid.

The form of the propeller has evolved substantially since the first successful aircraft. The combustion-based engines that are currently used to provide the shaft work to drive the propeller tend to get more efficient at larger scales. This has pushed aviation propeller design towards developing the most efficient means to absorb larger and larger amounts of power. As more shaft power is input, efficient propellers prefer a larger diameter. This is problematic when coupled with high rotational speeds (and forward flight velocity), as it yields tip speeds near or beyond the speed of sound. The losses experienced at the tips leads to a significant decrease in propulsive efficiency, as well as considerable noise. As a result, modern aircraft propellers constrain tip speed to avoid these compressibility-induced losses and acoustic concerns, instead opting for more blades, wider blade chords, or both. These highly-loaded blades compromise propulsive efficiency (thrust output vs. power input), but balance this through increased thermodynamic efficiency of the larger combustion-based powerplant.

Other issues enter the system-level trades for propellers as well. The cost and weight of combustion-based engines scales favorably at higher power levels, incentivizing fewer, higher-power engines vs. a greater number of lower-power engines. Also, powerplants that have a high number of moving parts (such as piston engines) suffer from maintainability and reliability concerns that are exacerbated as more engines are added to the vehicle. Hence, for a given size aircraft, the use of combustion-based engines pushes the designer to select the minimum number of powerplants possible, which forces propeller design into an exercise to absorb more and more power. Other than propulsive efficiency, the only other metric that suffers with fewer, higher-power propellers is the acoustic performance. Propellers exhibit poor acoustic performance at high tip speeds [1] and blade loadings, with limited shaping and shielding options available to stem the propagation of noise.

Finally, combustion-based engines are generally efficient at a narrow range of rotation speeds. Propellers, as rotating airfoils, are themselves only efficient over a narrow range of advance ratios, defined as a ratio of forward velocity to rotational speed. The advance ratio is related to the angle of attack seen at the local propeller blade airfoil section. As such, modern propellers are often designed with complicated mechanisms that maintain shaft speed within a small range and twist the blade to maintain an efficient angle of attack over a wide range of velocities. Such variable pitch mechanisms introduce additional complexity, cost, and weight into the system, along with associated reliability and maintainability penalties. 


\section{B. Electrically-Driven Propellers}

The use of electric motors as a source of shaft power provides an interesting opportunity for propeller design. Efficient electric motors, particularly those considered for aviation applications, have much higher power-to-weight ratios than their internal combustion alternatives, and exhibit fairly "scale free" behavior in terms of power vs. efficiency. Electric motors are exceptionally simple mechanical systems with, at most, a few moving parts. Hence, the cost, efficiency, reliability, and maintainability penalties associated with producing thrust from multiple propellers can be significantly lower than that seen with combustion-based engines.

As an added bonus, electric motors have relatively flat efficiency and torque curves over a range of rotational speeds. This can eliminate the need for complicated pitch change mechanisms for each propeller, and instead allows adjustment of motor/propeller rotational speed with forward flight speed to maintain an efficient advance ratio for the propeller. Ultimately, this further reduces the penalty associated with carrying multiple motors on electric aircraft. Such an operating approach has the secondary effect of reducing noise, since the times when the aircraft will be traveling slower (e.g. takeoff, climb, approach, and landing) will have to necessarily correlate to reduced propeller rotational speed.

The weight savings and efficiency gains of electrically-driven propellers are currently offset (and more) by the aircraft system-level implication of requiring a much heavier onboard energy storage system. Simply put, the energy density of batteries is much, much lower than the energy density of fuels used by combustion-powered concepts. Current technology limits for electrical energy storage and/or generation results in electric aircraft designs with poor range performance and low payload fractions. While significant research investments are being made into higherdensity electricity storage concepts, this remains a challenge for electrically-powered aircraft in the near-term. However, this concern is essentially independent of propeller sizing for electric aircraft.

\section{Distributed Electric Propulsion}

The characteristics of electric motors coupled with propellers enables new vehicle-level integration strategies that may otherwise soften the impact of low-energy density storage, largely by enabling a more efficient vehicle. One of the well-known tradeoffs in aircraft sizing is between an aircraft's stall speed and cruise speed. Lower stall speeds often necessitate a large wing, which is inefficient in cruise. Conversely, the high stall speeds of small, efficient wings for cruise require high takeoff and landing approach speeds, which affects everything from runway length to vehicle control. High-lift devices can make a smaller, cruise-efficient wing capable of higher lift at low speeds, but do so at increased complexity (with associated cost, maintainability, and reliability penalties). The size and cost of these mechanisms may even preclude their use on smaller aircraft. This is especially true on light aircraft that are representative of the On-Demand Mobility (ODM) paradigm, which are typically sized for single-digit passenger capacities.

Distributed Electric Propulsion (DEP) is a cornerstone technology for NASA's recent research into transformational mobility concepts such as ODM [2]. DEP uses a series of electrically-powered propellers along the leading edge of the wing to accelerate and turn the flow in an effort to scale the low-speed lift characteristics of the aircraft. This essentially treats these propellers as a different type of high-lift device. Additionally, wingtip-mounted propellers operate in the wingtip vortex to reduce induced drag and provide cruise thrust, while the leading edge high lift propellers fold back during cruise. NASA's "LEAPTech" DEP concept [3] is shown in Figure 1.

Properly designed, DEP enables design of aircraft with a cruise-sized (i.e. small) wings with adequate low-speed performance, without the need for the exotic high-lift devices seen on larger aircraft (slats with triple-slotted flaps, etc.), and in fact can yield a better maximum lift coefficient than such high-lift systems. The high power-to-weight ratio of the electric motors, along with the ability to utilize simple fixed-pitch propellers, greatly reduces the penalties associated with moving away from fewer propulsors. The exceptional reliability and maintainability of the propeller-motor system (due to the drastic reduction in mechanical complexity) further mitigates the otherwise prickly issues that can occur in these metrics when trading multiple, distributed propulsion systems vs. fewer, larger ones. Effectively, the leading edge propellers act as a simple, distributed high-lift device, and the tip propellers act as cruise propulsors. That is not to say that the leading edge propellers do not provide thrust; rather, they generate a thrust as a byproduct of accelerating the flow over the wing. 


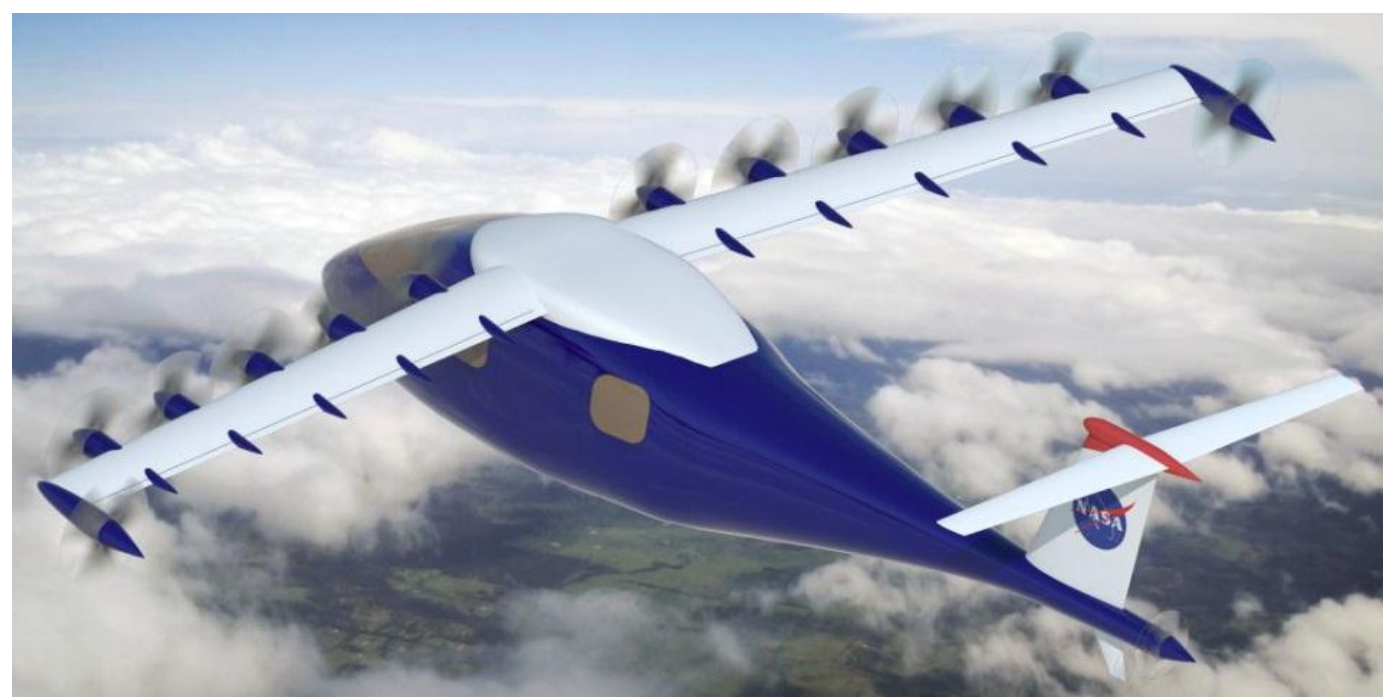

Figure 1. NASA LEAPTech Distributed Electric Propulsion concept [3].

\section{Approach}

Over the past few decades, the identification of propeller performance characteristics (and their impact on aircraft sizing) has largely fallen to semi-empirical methods, based on reduction of very large data sets to a few analytically-derived parameters used to describe propeller geometry. The method developed by Hamilton Standard in the 1960s [4] represents a well-known approach that has been widely updated and implemented in software $[5,6,7]$ by NASA for advanced propeller-driven concept studies.

The empirical-based methods relate propeller performance to a number of non-dimensional parameters. These include the power coefficient $C_{P}$, thrust coefficient $C_{T}$, advance ratio $J$, blade activity factor $A F$, and integrated design lift coefficient $C_{L_{i}}$. These terms are related to propeller geometric and operational parameters through the following relations:

$$
\begin{aligned}
& C_{P}=\frac{P}{\rho n^{3} D^{5}} \\
& C_{T}=\frac{T}{\rho n^{2} D^{4}} \\
& J=\frac{V}{n D} \\
& A F=\int_{x_{\text {hub }}}^{1} \frac{c(x)}{D} x^{3} d x \\
& C_{L_{i}}=4 \int_{x_{\text {hub }}}^{1} C_{L_{\text {des }}}(x) x^{3} d x
\end{aligned}
$$

where $T$ is propeller thrust, $\rho$ is the density of the fluid (air), $n$ is the rotational rate of the propeller, $D$ is the diameter of the propeller, $V$ is the freestream velocity, $c$ is the local section chord, and $C_{L_{\text {des }}}$ is the design lift coefficient of the local section. Note that the local section values ( $c$ and $C_{L_{d e s}}$ ) in Equations (4) and (5) are written as a function of the normalized blade span $x$, where $x$ is a ratio of radial blade station $r$ to propeller tip radius $R$ $\left(x=r / R\right.$ and $R=D / 2$ ). Equations (4) and (5) are defined from the normalized blade hub station $x_{h u b}$, which is taken from the Hamilton-Standard reference to be 0.15 for all of its propellers.

These coefficients and parameters are used to nondimensionalize a large body of empirical data for use in conceptual propeller performance estimation. The propellers contained within this empirical database were designed to be coupled with combustion-based engines as the source of shaft power, which inherently skews the dataset towards larger, higher RPM propellers for the reasons discussed in the previous section. Therefore, it is useful to understand the analytical underpinnings of propeller design based on more fundamental design parameters. 
Analytical propeller design methods for aircraft date back to the beginning of flight. The classical blade-element and momentum approaches are captured by Glauert [8], and have been updated to include numerical routines for design point and off-design performance $[9,10]$. These approaches can be used to parametrically evaluate minimuminduced loss propeller designs. However, using the minimum-induced loss concept applied to the design of propellers for electric aircraft has three drawbacks:

- The approach focuses on a single design point, rather than a spread of potential operating points. This assumes that the propeller will primarily operate at only a single condition. This is not a major issue as multipoint methods or constraint-based design can alleviate this concern.

- The approach assumes that an efficient propeller (one that uses the minimum amount of input shaft power per unit thrust) is best. Even when posed as a constrained optimization problem, this objective function does not properly account for some of the potential uses of a propeller as other than a thrust producing device. For example, the DEP technology discussed in the previous section effectively uses the leading edge propellers as high-lift devices by accelerating and turning the flow. Hence, it is more important to maximize induced velocity in the propeller slipstream (and not thrust) vs. minimum input power.

- The resulting blade geometries may not reflect other constraints, such as structural or manufacturing concerns. While the some issues can be addressed through integration of other analyses, manufacturing issues can be a significant constraint.

Despite these drawbacks, it is still possible to use analytical blade-element type approaches to design propellers. Instead of generating optimal designs, the off-design (analysis mode) capabilities of these approaches can be used to efficiently evaluate and explore a vast tradespace of propeller parameters.

\section{A. Propeller Design Parameters}

Propeller twist and chord profiles, like wing designs, are a compromise between strength and performance, plus a host of other concerns (e.g. acoustics, manufacturing, and cost). A propeller generates the majority of its thrust on the outer portion (towards the tip) of the blade due to the higher local velocity, which scales with $2 \pi r n$. Hence, a unit of chord at the outer portion (large $r$ ) will produce more lift (thrust) than one at the inner portion (small $r$ ). Thus, there is much less reason to have a substantial chord length near the propeller hub, and good reason to have more chord towards the tip. Similarly, the local induced angle of attack (due to the ratio of freestream to rotational velocity) is higher at the hub of the propeller and lower at the tips. This leads to the familiar twisted shape of the propeller blade, with large angles at the hub (root) and small angles at the tip.

Structural considerations are key as well. The bending of the blade under high loading leads to large relative thickness-to-chord values near the hub, and very thin sections near the tips. The latter (thin tips) also helps with higher-speed propellers (both in terms of flight velocity and rotational velocity due to high RPM), since the tips may approach or exceed the speed of sound. The trailing edge of propeller airfoils is often blunt to eliminate stress concentrations and premature cracking under load.

Other considerations cause variations in the otherwise familiar shape of propeller blades. The acoustic performance of the propeller can be drastically altered by reducing the tip speed (rotational velocity), which requires that each blade produces more thrust and absorbs more power at lower RPM. This often leads to larger-chord blades. Additionally, mild sweep back of the blade can reduce the onset of compressibility effects, which is beneficial from a performance and acoustics standpoint at the expense of greater manufacturing complexity and potential structural concerns. The increase in use of composite materials and computer-controlled manufacturing techniques has the potential to limit the effect of otherwise nontraditional designs.

For this paper, we consider a simple, smooth, continuous parametric representation of propeller design parameters for chord and twist distribution. This enables rapid exploration of the design space using relatively few parameters. In particular, four parameters are used to model chord $\left(y_{H}, y_{K}, y_{T}\right.$, and $\left.x_{H}\right)$, and three for twist $\left(\beta_{H}, \beta_{J}\right.$, and $\beta_{T}$ ), with second-order variation between the inflection points. These are defined in Figure 2. Note that airfoil selection is not currently considered, and a default symmetric airfoil is used in the analysis codes described later. Parameterization of airfoil sections is a planned future inclusion into this research. 

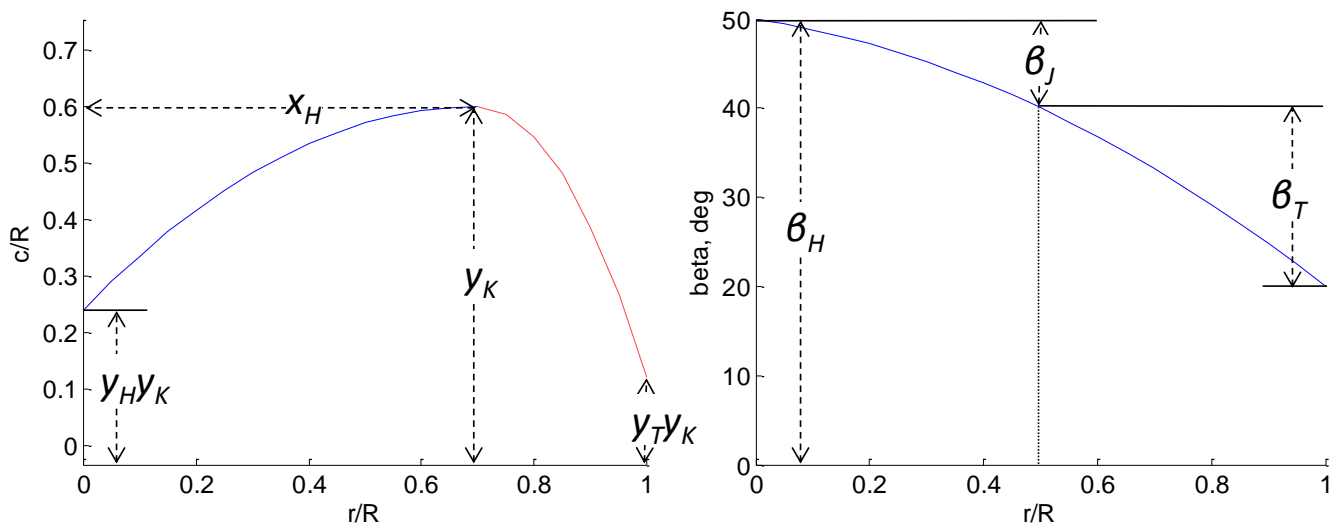

Figure 2. Propeller shape parameters for tradespace exploration.

The relationship of these parameters to the chord and twist distribution at any chordwise location $x$ is found by solving the following matrices:

$$
\begin{aligned}
& {\left[\begin{array}{ccc}
0 & 0 & 1 \\
x_{H}{ }^{2} & x_{H} & 1 \\
4 x_{H}{ }^{2} & 2 x_{H} & 1
\end{array}\right]\left[\begin{array}{l}
a_{H} \\
b_{H} \\
c_{H}
\end{array}\right]=\left[\begin{array}{c}
y_{H} y_{K} \\
y_{K} \\
y_{H} y_{K}
\end{array}\right]} \\
& {\left[\begin{array}{ccc}
\left(2 x_{H}-1\right)^{2} & 2 x_{H}-1 & 1 \\
x_{H}^{2} & x_{H} & 1 \\
1 & 1 & 1
\end{array}\right]\left[\begin{array}{l}
a_{T} \\
b_{T} \\
c_{T}
\end{array}\right]=\left[\begin{array}{c}
y_{T} y_{K} \\
y_{K} \\
y_{T} y_{K}
\end{array}\right]} \\
& {\left[\begin{array}{ccc}
0 & 0 & 1 \\
0.5^{2} & 0.5 & 1 \\
1 & 1 & 1
\end{array}\right]\left[\begin{array}{l}
a_{\beta} \\
b_{\beta} \\
c_{\beta}
\end{array}\right]=\left[\begin{array}{c}
\beta_{H} \\
\beta_{H}-\beta_{J} \\
\beta_{H}-\left(\beta_{J}+\beta_{T}\right)
\end{array}\right]}
\end{aligned}
$$

where $y=c / R, \beta$ is the local blade twist relative to the plane of propeller rotation, and $y_{H}, y_{K}, y_{T}, x_{H}, \beta_{H}, \beta_{J}$, and $\beta_{T}$ are as defined previously in Figure 2 . The coefficients $a_{H}, a_{T}, a_{\beta}, b_{H}, b_{T}, b_{\beta}, c_{H}, c_{T}$, and $c_{\beta}$ are used to form the second-order polynomials

$$
\begin{array}{ll}
y=a_{H} x^{2}+b_{H} x+c_{H} & x \leq x_{H}, \\
y=a_{T} x^{2}+b_{T} x+c_{T} & x>x_{H}, \text { and } \\
\beta=a_{\beta} x^{2}+b_{\beta} x+c_{\beta} & \forall x .
\end{array}
$$

These coefficients are found by solving Equations (6) through (8) in terms of $y_{H}, y_{K}, y_{T}, x_{H}, \beta_{H}, \beta_{J}$, and $\beta_{T}$.

The two operational parameters for the propeller are the freestream velocity and rotational speed. These will necessarily vary throughout the aircraft's flight envelope, but it is important to pick some reference conditions for comparison of different designs. For this paper, rotational tip speed $V_{t i p}$ will be used to define reference rotational speeds for various designs. Tip speed is related to propeller (shaft) rotation rate by

$$
V_{t i p}=\pi D n
$$

using the values defined earlier. Tip speed is a preferred design parameter to rotational speed as it provides a means to limit design combinations that may otherwise result in high compressibility losses (combinations of large diameters and high rotation rates), as well as a simple means to compare propeller acoustic performance (knowing that high tip speeds will come with significant acoustic penalties). For this initial exploration, the plane of propeller rotation is considered to be perpendicular to the aircraft velocity vector; that is, no aircraft angle of attack effects are considered. 


\section{B. Computational Approach}

The analytical methods described above are easily adaptable to computational analysis. This provides the added advantage of scripted tradespace exploration for a variety of discrete points, which enable diverse exploration of the propeller design space using the parameters described above. For this research, the authors adapted XROTOR [11] as a means to evaluate the designs, with $\operatorname{MATLAB}^{\circledR}$ [12] used to generate the design parameter combinations, script the appropriate XROTOR inputs, and parse the XROTOR output for post-processing.

$\mathrm{XROTOR}$ is an open-source design and analysis program for propellers. It can design minimum-induced loss rotors for a particular design point, as well as conduct off-design point analysis for a variety of operating conditions. It allows for input of arbitrary propeller geometries as well. Its execution time is very fast on modern desktop or laptop computers, often requiring only a fraction of a second to converge. Its menu-driven inputs and text-based output file options make it well-suited for scripting, which is an important consideration when analyzing a large combinatorial space of design parameters.

Custom MATLAB ${ }^{\circledR}$ functions were written to communicate with XROTOR in batch mode. First, an initial geometry file was created based on the geometric design parameters outlined in the previous section. This provided input chord and twist at a series of spanwise stations, as well as hub and tip diameter. The default symmetric airfoil section was used throughout the blade profile. Next, the propeller file was loaded into XROTOR and used in offdesign mode at a specified altitude, velocity, and tip speed (input as rotational rate). After the analysis, the results were parsed and post-processed. Overall, a function evaluation of a single design parameter combination took approximately half a second on a $2.5 \mathrm{GHz}$ laptop processor in a Windows 7 environment. Other than preallocation of large matrices, little was done to optimize the scripts and functions for speed.

Post-processing recorded 20 scalar values for each design parameter combination. This included the eight geometric parameters $\left(y_{H}, y_{K}, y_{T}, x_{H}, \beta_{H}, \beta_{J}, \beta_{T}\right.$, and $\left.D\right)$, three operational parameters $\left(V, V_{\text {tip }}\right.$, and $\left.n\right), A F$, and $C_{L_{i}}$. Additionally, the input altitude (used to set atmospheric parameters) and twist at $75 \%$ of the radius (a typical value used in empirical propeller analysis as the scalar "propeller pitch") were recorded amongst the inputs. The XROTOR outputs were rolled up into the power per unit propeller diameter $P / D$, thrust per unit propeller diameter $T / D$, propulsive efficiency $\eta$, average swirl component of the propeller slipstream $\bar{\theta}$, and average dynamic pressure ratio increase in the slipstream $\bar{q}_{s} / q$. The latter three quantities are defined by

$$
\begin{aligned}
\eta & =J \frac{C_{T}}{C_{P}}, \\
\bar{\theta} & =\tan ^{-1}\left(\frac{\Delta \bar{V}_{T}}{\bar{V}_{i}}\right), \text { and } \\
\frac{\bar{q}_{S}}{q} & =\frac{\bar{V}_{i}^{2}}{V^{2}}
\end{aligned}
$$

where

$$
\begin{aligned}
& \bar{V}_{i}=\sqrt{\left(V+\Delta \bar{V}_{A}\right)^{2}+\Delta \bar{V}_{T}^{2}}, \\
& \Delta \bar{V}_{T}=\int_{0}^{1} \Delta V_{T}(x) d x, \text { and } \\
& \Delta \bar{V}_{A}=\int_{0}^{1} \Delta V_{A}(x) d x .
\end{aligned}
$$

The values $\Delta V_{T}$ and $\Delta V_{A}$ are the changes in tangential and axial velocities from the flight velocity. These are output from XROTOR at radial stations from zero to the propeller tip radius. The average velocity changes are found from numerical integration of Equations (17) and (18). The average dynamic pressure ratio from Equation (15) and average swirl component of the slipstream from Equation (14) are important metrics for propellers meant to augment wing lift, while the propulsive efficiency in Equation (13) represents a more traditional metric for propellers designed for efficient cruise thrust.

The design space is explored using Latin hypercube sampling of the selected design parameters. MATLAB's Statistical Toolbox offers a Latin hypercube sampling routine [13] that was used in the design space explorations for this paper. The function is used in space-filling mode, meaning that for a given number of parameters, the selected points attempt to fill the space by maximizing the distance from the other selected point. 
The purpose of this design exploration is to determine trends and patterns that may exist for propeller design related to electric propulsion concepts, including DEP. However, without a specific set of requirements applicable to an aircraft and mission, the tradespace cannot be adequately constrained to the point where only a select portion of the space appears favorable. Therefore, some means is necessary to reduce the otherwise large number of design options into a meaningful set of trades based on the output data. One method is to develop a composite objective function that merges the output data into a set of data that can be ordered and ranked.

Compromise Programming is a multi-objective technique that is a combination of Multi-objective Linear Programming and Goal Programming [14]. A popular method for Compromise Programming uses the bounds of the tradespace to normalize the data and has an objective function of the form [15]:

$$
\min F=\left\{\sum_{i=1}^{m}\left[\frac{w_{i}\left(F_{i}(\vec{v})-F_{i}^{*}\right)}{F_{i}^{-}-F_{i}^{*}}\right]^{p}\right\}^{1 / p}
$$

where $F$ is the composite objective function to be minimized, $w_{i}$ is the relative importance ("weight") of the $i^{\text {th }}$ objective (of $m$ objectives), $F_{i}(\vec{v})$ is the value of the $i^{\text {th }}$ objective for the given design parameter set $\vec{v}, F_{i}^{*}$ is the best value of the $i^{\text {th }}$ objective (either some nominal or the single-objective best amongst design space), $F_{i}^{-}$is the worst value of the $i^{\text {th }}$ objective, and $p$ is a parameter dependent on the type of norm to be used in the ranking. The most common values for $p$ are 1,2 , and $\infty$. When $p=1$, the approach is essentially a weighted sum. When $p=2$, the solutions are ranked based on minimum Euclidean distance away from the locus of best single-objective best values (the "ideal solution"). When $p=\infty$, the method will favorably rank solutions to minimize the maximum deviation from the locus of best single-objective best values. Any value of $p$ other than these three values will exhibit some combination of behaviors between these three break points $(p=1,2$, or $\infty)$, though since it is often not practical for computer programs to evaluate infinity, the results are practically the same for large values of $p$ (say, 》10).

As with most multi-objective techniques, using Compromise Programming to rank designs requires selection of weighting factors $w_{i}$ for each objective to describe the relative utility of one objective vs. another. This can lead to drastic differences in rankings, which may not yield meaningful information. To deal with this, this paper considers the probabilistic variation of relative importance values as discussed in [16], and ranks solutions by the number of times the resulting rank $F$ of a particular design $\vec{v}^{*}$ appears amongst the top 10\% of rankings within the tradespace. The robustness of solution rank to uncertainty in weighting factors is a first-order indicator of a desirable combination of design parameters.

\section{Results and Discussion}

The NASA LEAPTech DEP concept [3] utilizes two different types of electric propulsors within its architecture. These include the leading edge high-lift propellers and the wingtip-mounted cruise propellers. Each needs to be optimized considering local geometric and design constraints, and represent highly coupled multidisciplinary design analysis and optimization problems [17]. The broader tradespace implications of design of propellers for LEAPTech-type vehicles are described below.

\section{A. High-Lift Propellers}

The leading edge high-lift propellers are one of the key enablers for DEP technology. These propellers enable the use of a "cruise-sized" wing; that is, a wing with an optimum lift-to-drag ratio at a high cruise speed and altitude, by augmenting the lift over the wing at lower speeds. This lift augmentation comes in the form of artificially increased dynamic pressure over the wing by accelerating the flow beyond aircraft flight velocity using the slipstream produced by the leading edge propellers. Hence, the most important factor for high-lift propellers is not that they produce thrust efficiently, but rather, that they induce a high slipstream (increase in local velocity). This is at odds with typical propeller design, which defines propulsive efficiency as a measure of thrust production vs. input power, as seen in Equation (13). In the case of the leading edge high-lift propellers, efficiency is really a measure of lift augmentation vs. input power. This is a major reason why the metrics developed in the previous section consider the dynamic pressure ratio seen in Equation (15) - this is a measure of the average increase in lift potential across the propeller slipstream.

Unlike a single propeller, the leading edge propellers need to consider the efficiency with respect to the total input power across all propellers. Since the lift augmentation only occurs within the propeller slipstream, the DEP leading edge propellers are distributed across much, if not all of the leading edge of the wing to provide maximum lift augmentation. The metrics developed in the previous section consider $P / D$ rather than simply input power, since 
$P / D$ can be multiplied by the blown wingspan of the aircraft concept to get an estimate of total input power. Hence, the dynamic pressure ratio $\bar{q}_{s} / q$ vs. $P / D$ provides a better measure of leading edge propeller efficiency.

A vexing problem persists with the use of propellers to augment wing dynamic pressure at low speeds. The rotational motion of the propeller induces a tangential component of velocity, or "swirl," that can change the local angle of attack of the wing. This average swirl component $\bar{\theta}$, defined in Equation (14), represents the average change in local angle of attack the wing will see across the propeller slipstream. Even small changes in angle of attack can make the difference between large lift and stall, so it needs to be included as a metric within the leading edge high-lift propeller tradespace. In general, lower swirl values are considered desirable.

The initial look at this tradespace considers notional values for DEP-enabled concepts [2]. The propellers are limited to three blades and $450 \mathrm{ft} / \mathrm{sec}$ tip speed to dramatically lower the acoustic signature (one of the stated DEP benefits), and the evaluations consider a sea level, standard day with an aircraft at flight velocities of 30.5, 61, and 79.3 knots. The 61-knot speed represents the maximum stall speed for most light single-engine aircraft certified under 14 CFR Part 23, and is one of the limiting factors that leads to the compromise between low-speed capability and high-speed cruise on light general aviation aircraft. Since the "cruise-sized" wing is a stated DEP benefit, the desire for maximum lift at the single-engine stall speed provides an appropriate basis of comparison. The 30.5-knot speed is half of the stall speed, and represents an average value where lift augmentation will be needed during takeoff. Finally, the 79.3-knot speed is taken at 1.3 times the stall speed, and represents a typical approach speed of light aircraft. Overall, this is representative of a speed range where the leading edge high-lift propellers will need to be efficient. At higher speeds, the propellers can either be spun faster, or (preferably) turned off and folded back to maximize the cruise benefits of the cruise-sized wing. A constant hub diameter of $0.472 \mathrm{ft}$ is used for all calculations, as this is representative of the $144 \mathrm{~mm}$-diameter motor currently considered for the LEAPTech concept.

A Latin hypercube sample of the eight geometric design parameters $\left(y_{H}, y_{K}, y_{T}, x_{H}, \beta_{H}, \beta_{J}, \beta_{T}\right.$, and $\left.D\right)$ was created with 3000 different combinations, and evaluated for the three different flight velocity values. These variables, and their associated ranges, are given in Table 1.

Table 1. Design parameter ranges.

\begin{tabular}{|lll|}
\hline Variable & Low Value & High Value \\
\hline $\boldsymbol{y}_{\boldsymbol{H}}$ & 0.2 & 1.0 \\
$\boldsymbol{y}_{\boldsymbol{K}}$ & 0.2 & 0.8 \\
$\boldsymbol{y}_{\boldsymbol{T}}$ & 0.0 & 0.4 \\
$\boldsymbol{x}_{\boldsymbol{H}}$ & 0.2 & 0.8 \\
$\boldsymbol{\beta}_{\boldsymbol{H}}$ & $30 \mathrm{deg}$ & $60 \mathrm{deg}$ \\
$\boldsymbol{\beta}_{\boldsymbol{J}}$ & $5 \mathrm{deg}$ & $30 \mathrm{deg}$ \\
$\boldsymbol{\beta}_{\boldsymbol{T}}$ & $5 \mathrm{deg}$ & $30 \mathrm{deg}$ \\
$\boldsymbol{D}$ & $1 \mathrm{ft}$ & $3 \mathrm{ft}$ \\
\hline
\end{tabular}

The results with a $\bar{q}_{s} / q$ ratio of less than one at the 61-knot condition were filtered out, since these represented solutions with zero utility (no lift augmentation). After this filtering, 1571 designs remained in the space. Figure 3 shows the tradespace plot of the remaining designs for the critical 61-knot flight velocity, related to the wing stall condition.
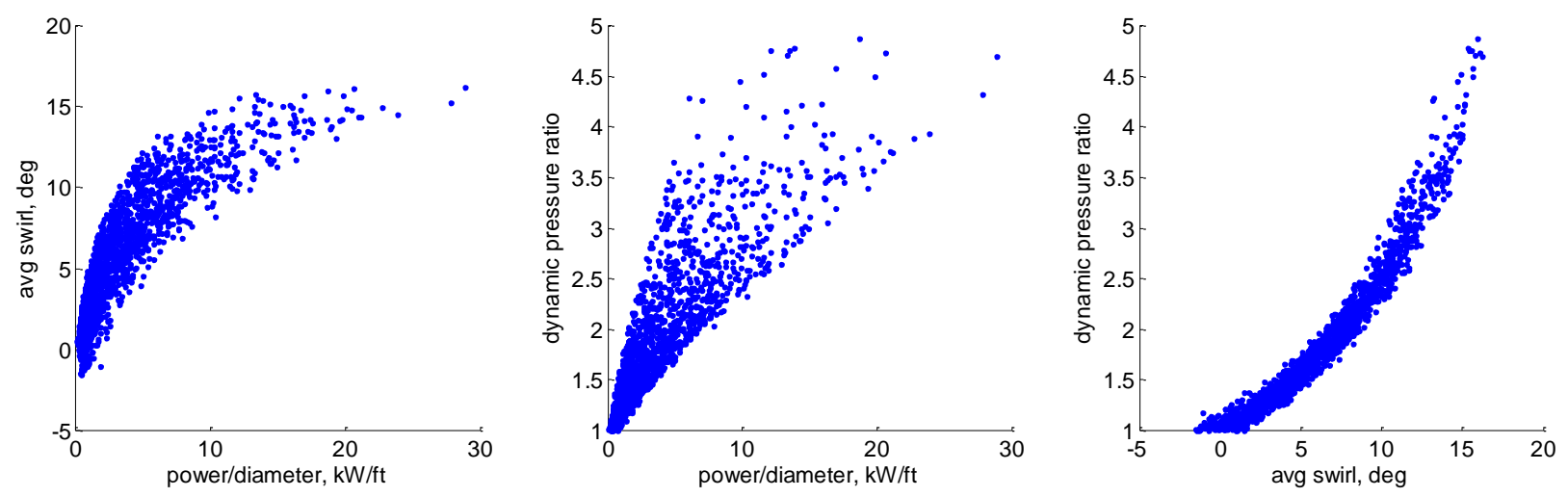

Figure 3. Tradespace plots for leading edge high-lift propellers at 61 knots. 
There are several trends seen within this plot. The practical limit on dynamic pressure at the 61-knot stall speed is slightly below 5 . This represents the "best" multiplier on lift relative to that achievable with the freestream velocity; however, this comes with very high power and swirl. Other notable trends are those that should be expected - higher power levels yield greater dynamic pressure ratios, but come at a higher cost in swirl. A promising trend is seen in the spread - there appears a set of designs with fairly low swirl, low input power, and high dynamic pressure ratio. The LEAPTech concept benefits from a dynamic pressure ratio of 2-3, so the expected average swirl per these results is between approximately 8-12 degrees, with power input per unit diameter of $2-15 \mathrm{~kW} / \mathrm{ft}$.

To gain a better understanding of the distribution of design parameters amongst the best designs, the 1571 remaining designs were evenly merged across all three speed ranges (that is, each design had its objective value at $30.5,61.0$, and 79.3 knots multiplied by $33 \%$ and added). The resulting values were ranked via the compromise programming method described earlier, with $p=2$. This involved another Latin hypercube space-filling variation of 3,000 combinations of weighting parameters for the three objectives. Of the 1571 designs, 58 were ranked in the top $10 \%$ at least $50 \%$ of the time. Given that the design variables are not especially intuitive, Figure 4 shows the variation of the propeller diameter and the three surrogate design parameters $\left(A F, C_{L_{i}}\right.$, and $\left.\beta_{3 / 4}\right)$ as histograms. The use of the surrogate design parameters helps to relate these propellers to the values cataloged in the empirical databases. The minimum and maximum ranges of the horizontal axes of these histograms represent the minimum and maximum values explored within the design, so they give a good sense of where the most favorable designs lie.
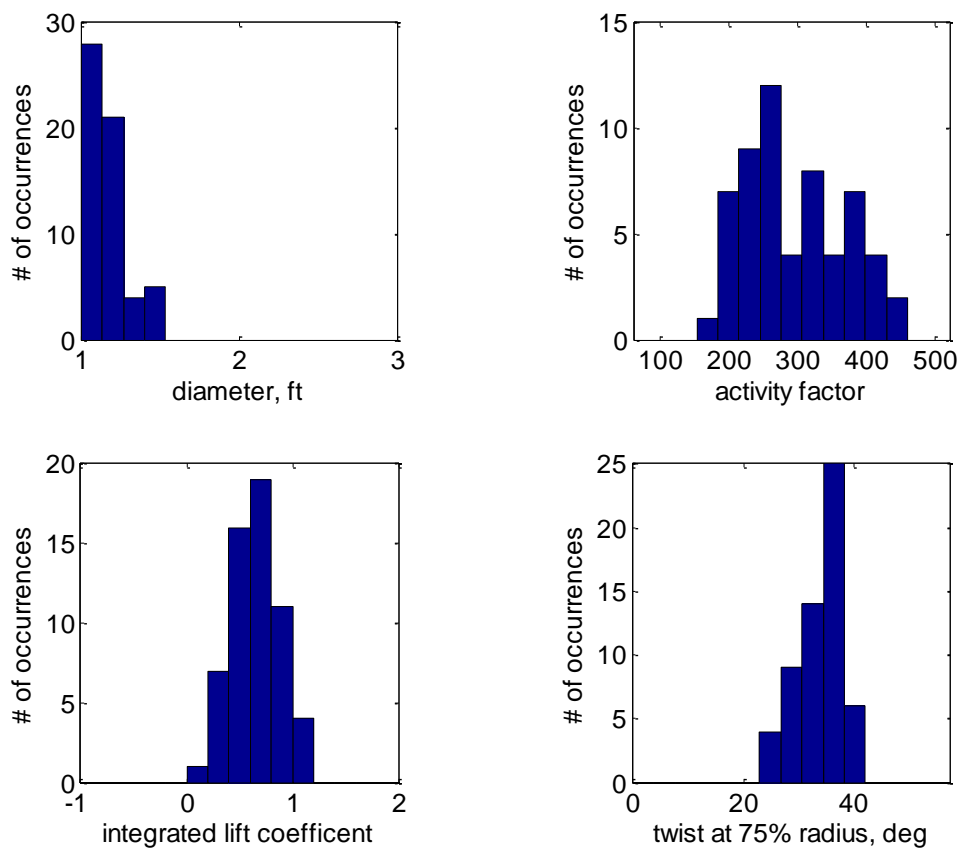

Figure 4. Variation in surrogate design parameters for best leading edge high-lift propellers.

One of the most striking results of this variation is the clustering of designs near the very low end of propeller diameter. After further introspection, this appears appropriate. At the first order, propeller thrust is due to a change in momentum - the product of mass flow through the propeller actuator disc and the induced velocity of the mass. As diameter is increased, thrust increases due to larger capture area (mass flow), and as pitch or blade chord is increased, thrust increases due to increased velocity. The high activity factors and twist at $3 / 4$ radius indicate that the favored designs are wide-chord, steeply-pitched propellers. This helps to provide high induced velocity without increasing the mass flow. That is, mass flow is proportional to area, and area is proportional to the square of the propeller diameter. Hence, the design does not favor large-diameter solutions. Future investigations may consider smaller-diameter propellers than those investigated here.

The favored designs have are biased to higher activity factors, which further indicates high solidity (large chord to diameter ratios). For reference, the empirical databases discussed earlier typically consider propellers with an activity factor of under 200, with a nominal value for a "regular" propeller around 100. Almost all of the favorable leading edge high-lift propellers had an activity factor of 200 or more. The integrated lift coefficients, which give an idea of the average section design lift coefficient, are normally distributed towards a mean of approximately 0.5 . However, this may be an artifact of the lack of airfoil selection within the initial design space exploration. 
Another way to view the output is to plot the chord and twist distributions of the 58 top recurring designs amongst the compromise programming ranking. These distributions are shown in Figure 5, with the chord station centered on the blade quarter chord. The chord vs. radial station values are shown approximately to scale.
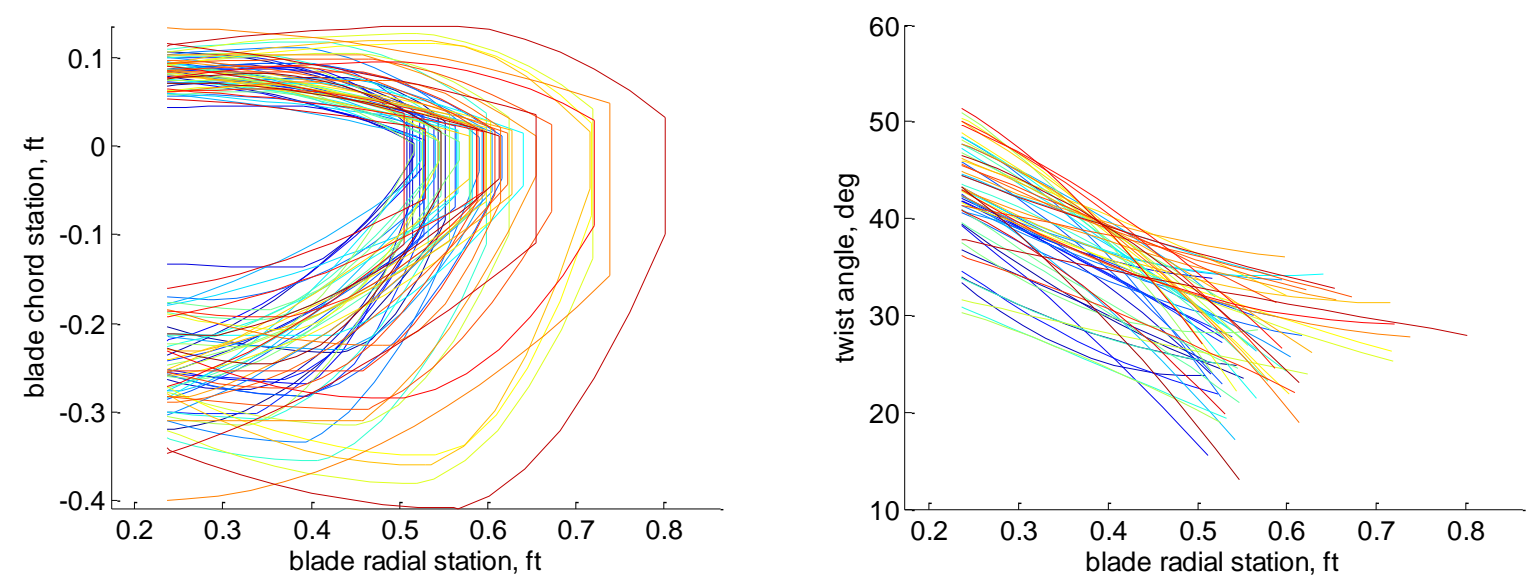

Figure 5. Chord (left) and twist (right) distributions vs. blade radial station for top 58 designs.

The resulting propeller chord distributions tend to take on a low-aspect ratio "paddle blade" shape rather than the high-aspect ratio shape of typical propellers. The small propeller radius is also apparent (recalling that the tip radii range from 0.5 to 1.5 feet). Furthermore, the twist distributions are fairly linear, though again this may be more of an artifact of airfoil selection (especially given the potential for changes in Reynolds number along the radius). The twist appears to shallow out for the larger radius designs, though this may again be slightly artificial due to the lack of airfoil selection. Finally, an inadequacy appears in the formulation of the chord and twist parameters developed in Equations (6) through (11); namely, the parameters should operate on the portion of the blade from the hub $(x=$ $\left.r_{h u b} / R\right)$ to tip $(x=1)$, rather than from $x=0$. This is because the parameter for the chord inflection point $x_{H}$ is occasionally less than the $x$ value of the hub, essentially destroying some available degrees of freedom in the design space exploration. Additionally, the blade twist angle at the root loses some meaning, since it represents a hub twist angle that really isn't located at the true hub, but further inward. Future studies will incorporate these changes.

The objective metrics distributions for the best propellers are shown in Figure 6. As with the previous figure, the horizontal axes represent the extreme values seen within the tradespace, to provide a sense of scale of the distribution of the best outputs. For clarity, these outputs are shown only for the 61-knot cases.
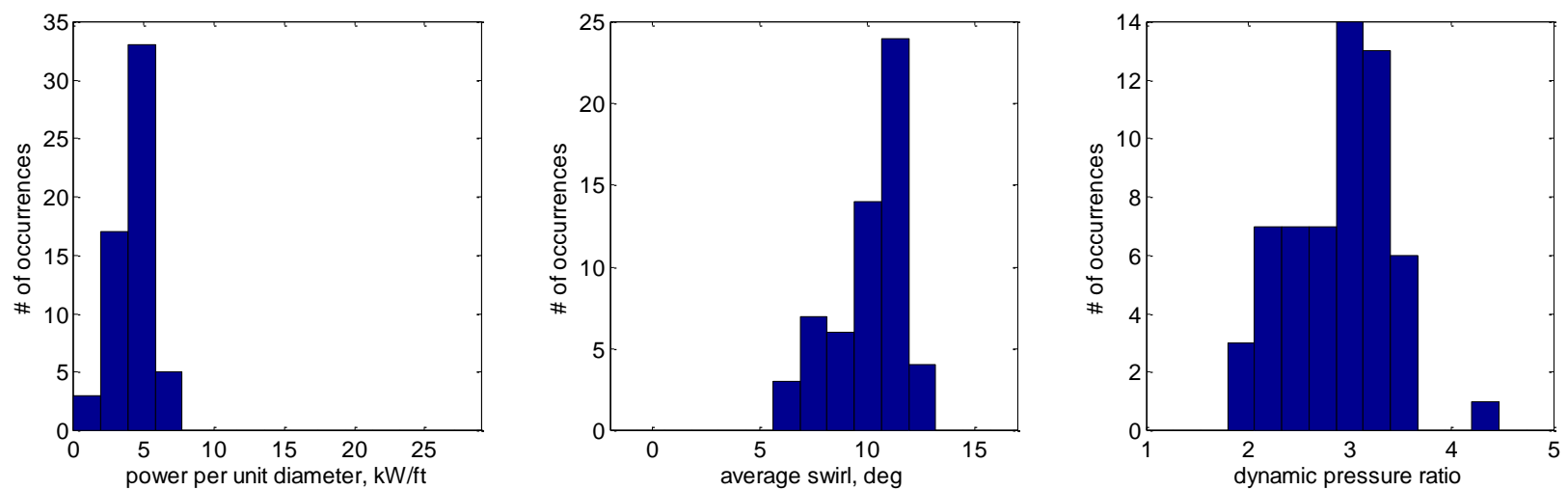

Figure 6. Variation in output parameters for best leading edge high-lift propellers at 61 knots.

These results are both encouraging and troubling for further exploration of DEP concepts. The average dynamic pressure ratio of the best concepts ranges from approximately 2-4, yielding potential for an exceptional reduction in wing area. This is tempered by the large average swirl, which will degrade the wing lift distribution. The favored propellers require about $5 \mathrm{~kW}$ of power per foot of diameter or less. Considering that the LEAPTech concept wing has less than $30 \mathrm{ft}$ of blown span, this results in fairly low power consumption penalties during high-lift operation (takeoff, climb, and approach). 


\section{Cruise Propellers}

The cruise propellers can use a more conventional design methodology. In this case, the minimum induced loss approach is more appropriate, since the vehicle will spend most of its time in cruise and will need to minimize the power input per unit thrust at high speed. However, the cruise propeller should be able to provide some thrust during lower-speed takeoff and climb. As mentioned earlier, one of the benefits of electric motors is that they can provide reasonably efficient power across a wide range of shaft rotation rates (RPM), which provides another, potentially less complex means of matching efficient angles of attack on the blade when compared to variable pitch or "constant speed" mechanisms. This fits well with reducing acoustic signature, since the lower flight velocities (such as those encountered during takeoff and climb) will require lower tip speeds if the relatively narrow efficient advance ratio range of fixed-pitch propellers is to be maintained. At higher flight velocities, the cruise propellers can exploit the benefit of higher speeds without worrying about annoying residents on the ground due to higher altitudes (though this does lead to increased potential for high cabin noise for the aircraft's occupants).

To evaluate this potential, we once again explore a design space to ensure adequate performance in cruise and at takeoff. The LEAPTech concept envisions a vehicle with a gross weight of around 3000-3500 pounds, and a lift-todrag ratio of 15-20 while cruising at an altitude of 12,000 ft and a velocity of 200 knots. This corresponds to a cruise thrust of 150-233 lbf, to be equally produced by the two wingtip propulsors (the leading edge propellers are turned off and folded back, but can be used for thrust at reduced cruise speeds as a contingency). An approximate upper bound is a propeller capable of $120 \mathrm{lbf}$ of thrust at the cruise condition.

For a minimum induced loss exploration, the design parameters are propeller diameter, propeller tip speed, and design lift coefficient. The ranges considered in this initial exploration are diameters between $2-4 \mathrm{ft}$, cruise tip speeds between 700-900 ft/sec, and blade design lift coefficients between 0.1-0.9. The metrics are cruise efficiency and takeoff thrust. The latter is evaluated at the 61-knot stall speed condition at sea level with tip speed constrained to $450 \mathrm{ft} / \mathrm{sec}$. For consistency with the designs explored earlier, only three-bladed solutions were explored, again with a constant hub diameter of $0.472 \mathrm{ft}$.

The MATLAB ${ }^{\circledR}$ scripts described earlier were modified to use XROTOR's minimum induced loss design feature for the cruise condition followed by the off-design evaluation at the stall/takeoff condition. Given the reduction in design variables, a 500-design Latin hypercube sample of the design space was taken. Figure 7 shows the resulting tradespace that exists between cruise efficiency and takeoff thrust.

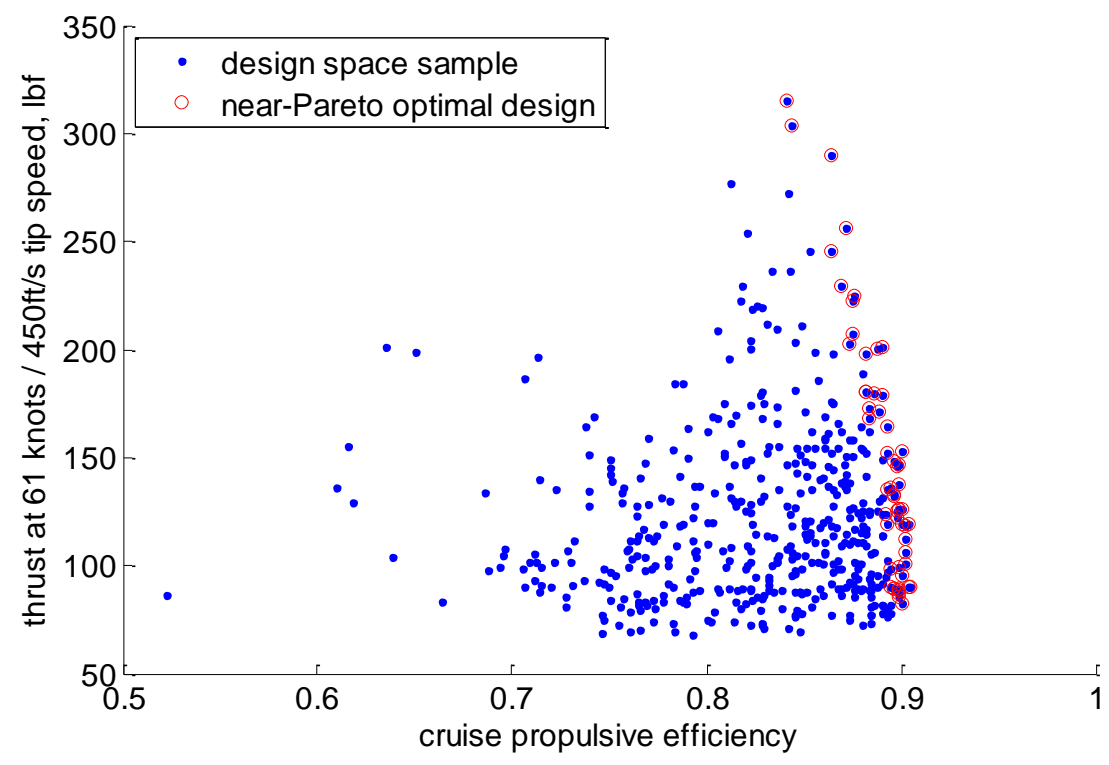

Figure 7. Cruise propeller tradespace exploration.

The near-Pareto optimal designs (defined as those that are not dominated by any design in more than one metric) in Figure 7 are highlighted with red circles. The term "near-Pareto" here means that the solutions may be slightly dominated in both dimensions (in this case no more than 1\%), which is used due to the discrete sampling technique. The resulting solutions show that a family of designs exists with the design space that are capable of providing high cruise efficiency (near 90\%), and still provide useful thrust at the takeoff/stall condition. The resulting distribution of design variables amongst these near-Pareto designs are given in Figure 8. 

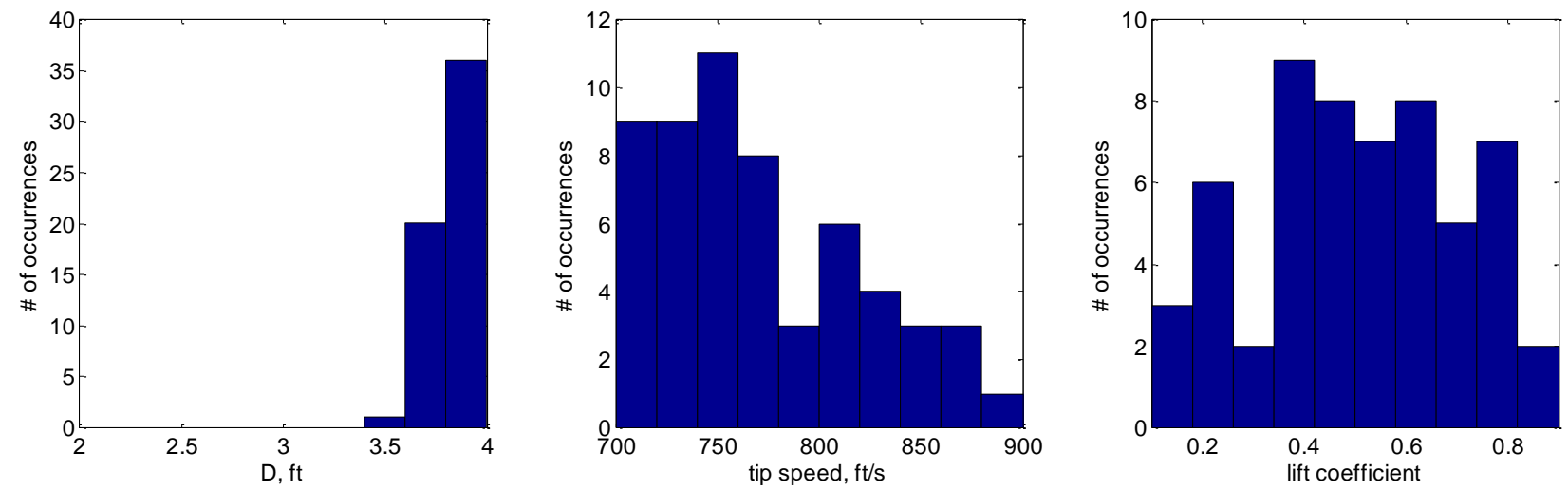

Figure 8. Variation in design parameters for near-Pareto-optimal cruise propellers.

Once again, the data presented in Figure 8 is scaled along the horizontal axis to the limits of the design space for the design parameters. The solutions strongly favor larger-diameter propellers, with a slight trend towards lower tip speeds. The design lift coefficients show no significantly discernable trend, but this may be due to the lack of any airfoil selection or definition. Additionally, the use of a lower-speed takeoff thrust condition (such as the 30.5-knot condition used for the leading edge high-lift propellers) may push the propeller towards lower design lift coefficients, since this would give additional stall margin at the lower speeds. To visualize the results further, the chord and twist profiles for the near-Pareto-optimal cruise propellers are shown in Figure 9.
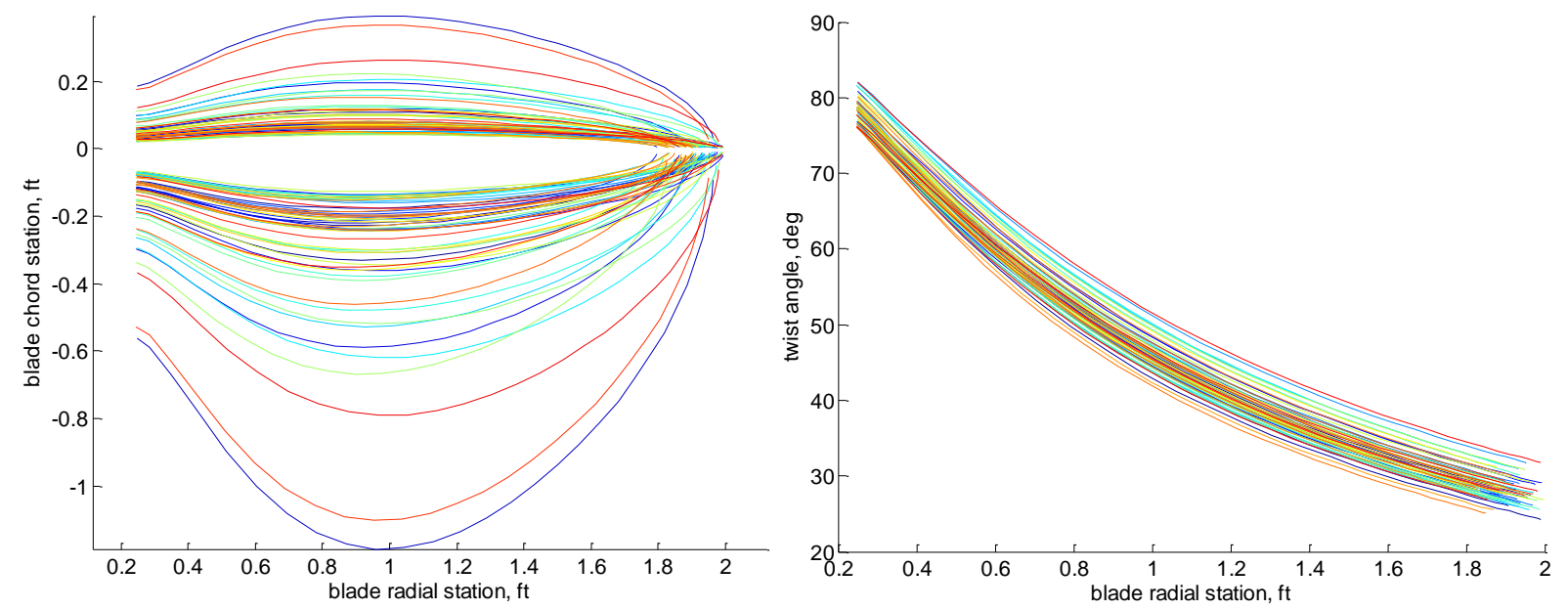

Figure 9. Chord (left) and twist (right) distributions vs. blade radial station for near-Pareto-optimal cruise propellers.

The blade profiles show a large variation in chord distribution and a fairly tight twist distributions. A few designs (those at the top end of the thrust spectrum from the near-Pareto optimal solutions in Figure 7) exhibit large-chord "paddle blade" designs, but for the most part the designs appear to be more conventional, narrow-chord (lower activity factor) blades as compared to those seen for the leading edge high-lift propellers from Figure 5. The ultimate selection of cruise propeller blades depends on the trade between high thrust performance during takeoff and cruise efficiency, but likely favors cruise efficiency given the amount of time the aircraft will spend in this condition.

\section{Conclusions}

Propellers coupled with electric motors are faced with a new, and different, set of degrees of freedom (as well as constraints) when compared to propellers designed for use with combustion-based engines. This is especially true of DEP-enabled aircraft concepts, where the leading edge propellers serve as a means to induce high velocities to turn and scale aerodynamic forces on the wing. This paper developed a method for evaluating and designing electricallydriven propellers, identified candidate metrics, and highlighted some of the salient trades and design choices for electrically-powered and DEP-enabled concepts. 
The method described in this paper enables the rapid design exploration of arbitrary rotors that can challenge current assumptions regarding efficient propeller design. This includes an approach for probabilistic ranking of concepts to otherwise unknown requirements. Explorations were conducted for a novel leading-edge propeller meant as a lift augmentation device through accelerated flow, rather than for thrust production. The results suggest that unconventional, high-solidity, low-diameter, high-pitch propellers are the most efficient means of augmenting the lift over the wing at low speeds. This comes at the price of increased swirl over the wing, which can cause significant variation in local angle of attack. This variation degrades the quality of the wing lift distribution, which could reduce the lift advantage of the accelerated flow. More detailed analysis and testing is needed to validate some of the assumptions for such augmented-lift concepts, which is the subject of future research and testing.

The design of electric propellers for high-speed cruise uses a more typical minimum-induced loss approach, but is modified to include moderate thrust production at low tip speeds to match advance ratio near the takeoff condition. This enables the cruise propellers to produce thrust at takeoff without the need for propeller pitch change mechanisms, with the added benefit of low noise due to reduced tip speed. The resulting propellers are capable of high cruise efficiency, while also providing significant thrust at lower speeds to assist with takeoff and climb.

Overall, this exploration was deliberately simplified to enable some initial discoveries and lend credence to analytical projections. Future work will greatly enhance this design and prediction capability, to include airfoil selection and propeller structural constraints. Additionally, this approach is being developed in parallel with exploratory acoustics models and integrated aero-propulsive analysis; later work will merge all of these into a larger tradespace exploration for concepts such as DEP. Ultimately, the goal is to validate this integrated approach with data from upcoming DEP ground tests. Properly validated, the integrated design approach will be used to aid the design of a DEP flight demonstrator.

\section{Acknowledgments}

This work was funded under the Aerosciences Project of the NASA Fundamental Aeronautics Program. The authors would like to thank Andrea Storch, Bill Fredericks, Jeff Viken, and Mark Guynn for their support, guidance, and leadership over the course of this project.

\section{References}

1. Anon., "Prediction Procedure for Near-Field and Far-Field Propeller Noise," AIR 1407, Society of Automotive Engineers, Inc., Warrendale, PA, May 1977.

2. M. Moore, K. Goodrich, J. Viken, J. Smith, B. Fredericks, T. Trani, J. Barraclough, B. German, M. Patterson, "High-Speed Mobility through On-Demand Aviation," AIAA 2013-4373, Aviation Technology, Integration, and Operations Conference, Los Angeles, CA, August 2013.

3. M. Moore and B. Fredericks, "Misconceptions of Electric Propulsion Aircraft and their Emergent Aviation Markets," AIAA 2014-0535, 52 ${ }^{\text {nd }}$ Aerospace Sciences Meeting, National Harbor, MD, January 2014.

4. Anon., "Generalized Method of Propeller Performance Estimation," PDB 6101, Revision A, Hamilton Standard Division of United Aircraft Corporation, June 1963.

5. R. Worobel, M. G. Mayo, “Advanced General Aviation Propeller Study,” NASA CR 114289, April 1971.

6. R. Worobel, M. G. Mayo, “Advanced General Aviation Propeller Study,” NASA CR 114399, December 1971

7. R. M. Plencner, P. Senty, T. J. Wickenheiser, "Propeller Performance and Weight Predictions Appended to the Navy/NASA Engine Program," NASA TM 83458, August 1983.

8. H. Glauert, “Airplane Propellers," Aerodynamic Theory (ed. by F. Durand), Div. L, Vol. 4, Julius Springer, Berlin, 1935, pp. 169-359.

9. E. E. Larrabee, "Practical Design of Minimum Induced Loss Propellers," SAE 790585, Business Aircraft Meeting and Exposition, Wichita, KS, April 1979.

10. C. N. Adkins, R. H. Liebeck, "Design of Optimum Propellers," Journal of Propulsion and Power, 10:5:676-682, 1994.

11. M. Drela, H. Youngren, "XROTOR Download Page,” http://web.mit.edu/drela/Public/web/xrotor/, accessed 6 May 2014.

12. "MATLAB - The Language of Technical Computing," http://www.mathworks.com/products/matlab/, accessed 6 May 2014.

13. "Latin hypercube sample - MATLAB lhsdesign," http://www.mathworks.com/help/stats/lhsdesign.html, accessed 6 May 2014.

14. M. Zeleny, Multiple Criteria Decision Making, McGraw-Hill, New York, NY, 1982.

15. G. N. Vanderplaats, Numerical Optimization Techniques for Engineering Design, Third Edition, Vanderplaats Research and Development, Inc., Colorado Springs, CO, 1999.

16. N. Borer, Decision Making Strategies for Probabilistic Aerospace Systems Design, Ph.D. Dissertation, Georgia Institute of Technology, Atlanta, GA, May 2006.

17. M. D. Patterson, M. J. Daskilewicz, B. J. German, "Distributed Propellers: Multidisciplinary Analysis Needs and Aerodynamic Modeling Development," AIAA 2014-0534, 52 ${ }^{\text {nd }}$ Aerospace Sciences Meeting, National Harbor, MD, January 2014. 